\title{
Antibiotic sensitivity profile of Klebsiella isolates and it's impact on clinical outcome
}

\author{
Rahul R. Damor, Amita R. Kubavat*
}

Department of Pharmacology, P. D. U. Government Medical College, Rajkot, Gujarat, India

Received: 23 May 2019

Revised:11 June 2019

Accepted: 06 July 2019

*Correspondence to:

Dr. Amita R. Kubavat,

Email: aminilu2000@

yahoo.com

Copyright: (C) the author(s), publisher and licensee Medip Academy. This is an openaccess article distributed under the terms of the Creative Commons Attribution NonCommercial License, which permits unrestricted noncommercial use, distribution, and reproduction in any medium, provided the original work is properly cited.

\begin{abstract}
Background: Antimicrobials are the greatest discovery of the twentieth century. To limit the emergence and spread of resistance, antibiotic therapy should be adjusted according to the results of microbiological culture. Klebsiella isolates causes various types of infections and the incidence of antibiotic resistance is also high in Klebsiella infections. So, Authors plan this study to analyze how the results of microbiological cultures influence the antibiotic use in the treatment of Klebsiella infections.

Methods: It is a record based observational prospective study which assessed the impact of Klebsiella positive culture results on antibiotic prescribing pattern and its impact on clinical outcome. Patients with empirical antibiotic therapy and Klebsiella positive were included and patients with inadequate data were excluded in this study and the data were recorded. Recorded data were entered and analyzed in Microsoft Office Excel-2013. Unpaired student t-test was used to compare the mean duration of hospital stay using Past software (version 3.20). Results: There were total 400 patients in our study. Amikacin was the most common drug $(n=202)$ used as empirical therapy. Empirical antibiotic therapy was changed in 161(40.25\%) patients. Meropenem was the most commonly used definitive drug. Mean duration of hospital stay is less in empirical sensitive antibiotic therapy as compared to empirical resistance antibiotic therapy having $\mathrm{p}$ value $<0.0001$ which shows significant difference between two groups showing better clinical outcome.
\end{abstract}

Conclusion: Initial empirical therapy with broad-spectrum antimicrobials is a treatment strategy for severe Klebsiella infections.

Keywords: Antibiogram, Definitive therapy, Empirical therapy, Klebsiella isolates, Prescription pattern, Resistance, Sensitivity

\section{INTRODUCTION}

Antimicrobials are the greatest discovery of the twentieth century. Antimicrobial agents are considered as "miracle drugs" that are our leading weapons in the treatment of infectious disease. In the pre-antimicrobial era, infectious diseases accounted for significant morbidity and mortality. All this changed with the use of antimicrobial agents and the 'golden era of antimicrobials' saw a dramatic fall in the mortality due to infections. This miracle seems to be short lived as there is irresponsible and erratic use of these life- saving drugs has resulted in the emergence of drug resistance in many organisms and morbidity and mortality due to hospital acquired infections is on the rise. In June 2000, WHO issued warning that the widespread use of antimicrobials to treat diseases is producing increasingly drug resistant infections all over the world. ${ }^{1}$

The appropriate use of antibiotics is an important aspect in the treatment of infections. Antibiotics are frequently prescribed because of clinical suspicion of infection while the results of microbiological analysis are still awaited. 
Early clinical recognition of infection, rapid laboratory detection of the causative organisms and prompt initiation of appropriate antimicrobial therapy are all essential aspects of the management of severe infections. When the pathogens and their susceptibilities are determined, streamlining and adapting antimicrobial regimens are important to ensure optimal treatment and to limit the untoward consequences of the misuse of antimicrobial agents particularly the selection of resistant microorganisms and excessive cost of treatment. ${ }^{2}$

Several strategies have been suggested to improve antibiotic prescribing such as surveillance of antibiotic resistance, monitoring and auditing of antibiotics use, the use of consensus guidelines or computer decision support and improving multidisciplinary cooperation. ${ }^{3}$ A microbiological culture is a method of multiplying microbial organisms by letting them reproduce in predetermined culture media under controlled laboratory conditions. They are used to determine the type of organism, its abundance in sample or both. It is one of the primary diagnostic methods of microbiology and act as a tool to determine the cause of infectious disease. ${ }^{4}$

Empirical antibiotic therapy may be influenced by microbiological culture results either by discontinuation of therapy in case of negative cultures or by broadening or narrowing of spectrum of antibiotics. To limit the emergence and spread of resistance, antibiotic therapy should be adjusted according to the results of microbiological culture. ${ }^{5}$ The results of microbiological cultures are often ignored because the patients are doing well on empiric therapy. Although microbiological studies form the basis of effective treatment, clinicians and hospital services show varying degree of interest in microbiological analysis to decide appropriate treatment. 6,7

There is increased case of Klebsiella infection and due to that there may be increased incidence of antibiotic resistance. Klebsiella causes various types of infection like: urinary tract infections, nosocomial infections, wound infections, community acquired pneumonia and chronic genital ulcerative disease etc. The rate of resistance of Klebsiella isolates to different antibiotics is increased day by day. In recent years, Klebsiella have become important pathogens in nosocomial infections. The importance of Klebsiella in the ever increasing number of gram negative aerobic bacillary nosocomial infections in the India has been well documented. Epidemic and endemic nosocomial infections caused by Klebsiella species are leading causes of morbidity and mortality. ${ }^{8}$

If Authors don't take immediate action then only handful antibiotics will be left to cure diseases. Multidrug resistant bacteria cause serious nosocomial and community acquired infections that are hard to eradicate by using available antibiotics. Moreover, extensive use of broadspectrum antibiotics in hospitalized patients has led to increased prevalence of Klebsiella isolates as well as development of multidrug resistant strains of Klebsiella. So, by reducing these type of resistance by knowing the sensitivity pattern we can overcome the multi drug resistance. ${ }^{9}$

Although appropriateness of antibiotic prescribing has been investigated frequently, the impact of microbiological culture results on antibiotic prescribing has not been analyzed frequently so this study is designed to analyze how the results of microbiological cultures influence the antibiotic use in the treatment of infection. The aim of the study was to assess the antibiotic sensitivity and resistance pattern of Klebsiella isolates. The primary objective of the study was to know the antibiotic prescription pattern for patients with Klebsiella infection and to know the duration of hospital stay of antibiotic sensitive and antibiotic resistance cases for measuring clinical outcome. The secondary objective of the study was to know the burden of disease caused by the Klebsiella isolates.

\section{METHODS}

It is a record based observational prospective study which assessed the impact of Klebsiella positive culture results on antibiotic prescribing pattern and its impact on clinical outcome. The study was conducted at a tertiary care hospital attached to government medical college. The study was conducted for the duration of 18 months i.e. from October 2016 to March 2018. Patients who were on empirical antibiotic therapy and culture positive for Klebsiella pneumoniae, Patients of either sex of any age admitted in the wards of hospital were included in the study. Patients with inadequate case record or data, discharged against medical advice and those not willing to participate in the study were excluded. Total 400 patients were included in the whole duration of the study. The prior permission of Institutional Ethics Committee was taken.

The patient's demographic data, clinical data and therapeutic data were collected and recorded. Data of the patients matching inclusion criteria were only recorded. Before collection of the data informed written consent of the patients was taken. The patients were also provided the patient information sheet to understand the study procedure. No intervention was done in the treatment of the patients by the investigator.

Data were analyzed as: age wise distribution, gender wise distribution, department wise distribution, distribution of patients according to diagnosis, distribution of patients according to type of collected specimen, empirical antibiotic therapy according to antimicrobial drugs, change in the antibiotic therapy after culture sensitivity testing, definitive antibiotic therapy according to diagnosis, definitive antibiotic therapy according to antimicrobial drugs, mean duration of stay in empirical sensitive and empirical resistance therapy group, antibiogram of microorganisms. 


\section{Statistical analysis}

Recorded data were entered and analyzed in Microsoft Office Excel-2013 and using descriptive statistics. Unpaired student t-test was used to compare the mean duration of hospital stay using Past software (version 3.20). Wherever necessary, the results were depicted in the form of percentages and graphs..

\section{RESULTS}

This prospective, observational study was carried out to assess the impact of Klebsiella pneumoniae positive culture on antibiotic prescribing pattern at a tertiary care hospital. The study was conducted on 400 patients over a period of 18 months. The relevant data were collected and analyzed as follows. In this study, out of 400 patients, majority of patients i.e. $220(55 \%)$ were in 1 to 15 years of age group followed by $66(16.5 \%)$ patients were of age 31 to 45 years. Patients being enrolled in the study were ranging from day- 1 to 81 years old. So, the pediatrics patients showing the maximum number of patients load. Out of 400 patients enrolled in the study, $226(56.5 \%)$ patients were male and $174(43.5 \%)$ patients were female. During the study, patients admitted in the different departments of government hospital were enrolled. Pediatrics department showing the maximum number of patients of $217(54.25 \%)$ from all the department. During the study, patients with different 32 diagnosis were enrolled out of which Neonatal sepsis $(n=82,20.5 \%)$ was the most common diagnosis. During the study 7 types of specimens have been collected with Klebsiella isolates in 400 patients. Blood was the most common specimen $(n=226,56.5 \%)$ of all the specimens.

Table 1: Empirical Antibiotic Therapy According to Antimicrobial Drugs.

\begin{tabular}{|c|c|c|c|}
\hline Antimicrobial Group & Name of drug & $\begin{array}{l}\text { No. of patients } \\
\text { receiving drugs }\end{array}$ & $\begin{array}{l}\text { Total no. of } \\
\text { patients }\end{array}$ \\
\hline \multirow{4}{*}{ Penicillins } & Augmentin & 17 & \multirow{4}{*}{216} \\
\hline & Ampicillin & 1 & \\
\hline & Ampicillin/sulbactam & 118 & \\
\hline & Piperacillin/Tazobactum & 80 & \\
\hline \multirow[b]{2}{*}{ Aminoglycosides } & Amikacin & 202 & \multirow{2}{*}{215} \\
\hline & Gentamicin & 13 & \\
\hline \multirow{4}{*}{ Cephalosporins } & Ceftriaxone & 91 & \multirow{4}{*}{139} \\
\hline & Cefotaxime & 46 & \\
\hline & Cefuroxime & 1 & \\
\hline & Cefixime & 1 & \\
\hline \multirow{3}{*}{ Fluoroquinolones } & Norfloxacin & 11 & \multirow{3}{*}{81} \\
\hline & Ciprofloxacin & 6 & \\
\hline & Levofloxacin & 64 & \\
\hline Carbapenems & Meropenem & 37 & 37 \\
\hline Nitroimidazoles & Metronidazole & 32 & 32 \\
\hline Lincosamide & Clindamycin & 5 & 5 \\
\hline Oxazolidinone & Linezolid & 5 & 5 \\
\hline Azoles & Fluconazole & 5 & 5 \\
\hline Glycopeptides & Vancomycin & 4 & 4 \\
\hline Macrolides & Azithromycin & 3 & 3 \\
\hline Polypeptide & Colistin & 3 & 3 \\
\hline
\end{tabular}

As shown in Table 1, Penicillin group $(n=216)$ followed by Aminoglycosides group $(n=215)$ of antimicrobial drugs were most commonly used as an empirical antibiotic therapy. Amikacin was the most common drug $(n=202)$ used as empirical therapy.

Out of 400 patients, $80(20 \%)$ patients received one antimicrobial drug, $295(73.75 \%)$ patients received two antimicrobial drugs and $25(6.25 \%)$ patients received three antimicrobial drugs.
Table 2: Empirical antibiotic therapy according to antimicrobial drugs.

\begin{tabular}{|ll|}
$\begin{array}{l}\text { Change in Antibiotic } \\
\text { Therapy }\end{array}$ & $\begin{array}{l}\text { No. of Patients } \\
(\mathbf{N}=400)\end{array}$ \\
\hline Yes & $161(40.25)$ \\
\hline No & $239(59.75 \%)$ \\
\hline
\end{tabular}

Out of 400 patients, empirical antibiotic therapy has been changed to definitive antibiotic therapy in $161(40.25 \%)$ 
patients after culture sensitivity testing which is shown in Table 2. As shown in Table-3, Carbapenems like Meropenem was the most commonly used drug in definitive antibiotic therapy. Out of 161 patients receiving the definitive antibiotic therapy, $58(36.02 \%)$ patients received one antimicrobial drug, $103(63.98 \%)$ patients received two antimicrobial drugs.

Table 3: Definitive Antibiotic Therapy According to Antimicrobial Drugs.

\begin{tabular}{|llll|}
\hline $\begin{array}{l}\text { Antimicrobial } \\
\text { Group }\end{array}$ & Name of Drug & No. of patients receiving the drugs & Total No. of Patients \\
\hline Carbapenems & Meropenem & 85 & 85 \\
\hline \multirow{3}{*}{ Fluoroquinolones } & Norfloxacin & 2 & 75 \\
\cline { 2 - 4 } & Ciprofloxacin & 2 & \multirow{2}{*}{54} \\
\cline { 2 - 4 } Levofloxacin & 71 & 21 \\
\hline Penicillins & Augmentin & 16 & 14 \\
\hline Urinoglycosides & Piperacillin/Tazobactum & 38 & 6 \\
\hline Macrolides & Amikacin & 21 & 4 \\
\hline Nitroimidazoles & Aitrofurantoin & 14 & 2 \\
\hline Glycopeptides & Metronidazole & 6 & 2 \\
\hline Oxazolidinone & Linezolid & 2 & 1 \\
\hline Cephalosporins & Cefotaxime & 2 & \\
\hline
\end{tabular}

Table 4: Duration of Stay in Empirical Sensitive and Empirical Resistance Antibiotic Therapy group.

\begin{tabular}{|lll|}
\hline Group & Duration of hospital stay in days $($ mean \pm SD $)$ & Number of patients $(\mathbf{n}=\mathbf{4 0 0})$ \\
\hline Empirical sensitive & $8.87 \pm 4.51$ & 239 \\
\hline Empirical resistance & $11.89 \pm 5.10$ & 161 \\
\hline
\end{tabular}

As shown in Table 4, mean duration of stay in empirical sensitive therapy group and empirical resistance therapy group were $8.87 \pm 4.51$ days and $11.89 \pm 5.10$ days respectively. So, there was highly significant difference between two treatment arms on decreasing duration of hospital stay according to diagnosis (unpaired t-test; $\mathrm{P}$
$<0.0001)$. During the study antibiotic culture sensitivity testing of Klebsiella isolates was carried out. Figure 1 shows the antibiogram of Klebsiella pneumoniae in which total number of drugs tested of different groups and its sensitivity was recorded. Carbapenem like Meropenem shows $100 \%$ of sensitivity while the Cephalosporins shows the minimum number of sensitivities of $20 \%$.

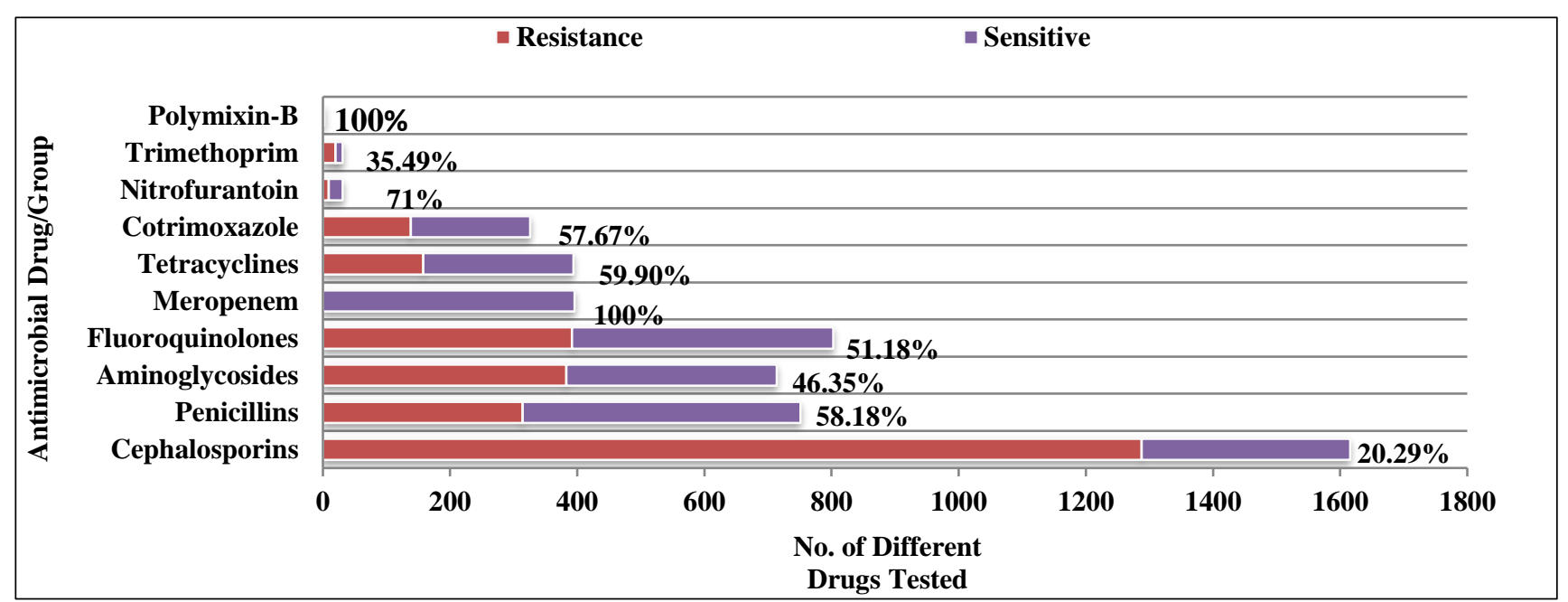

Figure 1: Antibiogram of Klebsiella isolates showing Sensitivity. 


\section{DISCUSSION}

This is prospective, observational study was carried out to assess the impact of Klebsiella Pneumoniae positive culture results on antibiotic prescribing pattern and resistance pattern and its effect on clinical outcome on the basis of duration of hospital stay. It has been demonstrated that adjusting antibiotic therapy according to the results of microbiological culture findings leads to a narrowing of antibiotic therapy. Thereby, the emergence of antibiotic resistance can be avoided or delayed. ${ }^{10}$ Numerous studies of patients with a variety of serious infections have shown that initiating inappropriate empirical antimicrobial therapy or delaying the initiation of appropriate antimicrobial therapy is associated with increased mortality. ${ }^{11,12}$

Klebsiella is well known to most clinicians as a cause of community-acquired bacterial pneumonia, sepsis, urinary tract infection which has a high fatality rate if untreated and are associated with hospitalization. ${ }^{13}$ In our study majority of the patients were lies below 1 month of age i.e. 171 $(42.75 \%)$ with highest number of patients from Pediatrics department. A study done by Fahmey S. et al shows similar results. ${ }^{14}$ The most common diagnosis were Neonatal Sepsis, Pneumonitis, Urinary Tract Infection etc. respectively.

In this study, Penicillins (54\%) like Ampicillin/Sulbactam and Piperacillin-Tazobactum, and Aminoglycoside $(53.75 \%)$ antimicrobial agent like Amikacin were most commonly used drugs as an empirical antibiotic therapy in our study. The study done by Paterson et al and Tangden T. et al favours this use. ${ }^{15}$

During the study Neonatal Sepsis, Low Birth Weight with Sepsis cases $(n=126)$ were noted. Out of 126 cases 59 cases had changed empirical therapy after culture sensitivity testing. These patients were given either Ampicillin/Sulbactam and Amikacin or PiperacillinTazobactam and Levofloxacin as an empirical antibiotic therapy. After sensitivity testing the resistant antibiotics were changed to Meropenem and Levofloxacin as definitive therapy. Study performed by Suryawanshi et al shows similar pattern of antimicrobial usage in Neonatal Sepsis cases. ${ }^{16}$

During the study Ceftriaxone and amikacin were used as an empirical antibiotic therapy for the management pulmonary infections $(n=32)$ like Pneumonitis. Out of 32 cases 14 cases had changed empirical therapy after culture sensitivity testing to Amoxicillin-Clavulanic acid and Azithromycin as definitive antibiotic therapy. Study performed by Donovan et al supports the use of similar antimicrobial agents for the treatment of pulmonary infections. ${ }^{17}$ Antimicrobial agents like Norfloxacin or Amikacin along with Ceftriaxone were most commonly used as an empirical antibiotic therapy in Urinary Tract Infection patients $(\mathrm{n}=29)$. Out of 29 cases 20 cases had changed empirical therapy to Nitrofurantoin and
Levofoxacin after culture sensitivity testing. Study performed by Bouamri et al also shows similar results. ${ }^{18}$

The antibiogram of our study showed that Cephalosporins specially third generation showed $79.71 \%$ of resistance which is highest of all is a growing concern for increasing resistance.15 A study done by Park S. et at also showed similar profile of resistance for third generation Cephalosporins. ${ }^{19}$ There was highest sensitivity seen with Carbapenems like Meropenem 100\% in the antibiogram of the study. The study done by Leisy Azar S. et al also showed the similar pattern for the Carbapenems. ${ }^{20}$ In authors study, antibiotic prescribing was influenced only in $161(40.25 \%)$ out of 400 patients with positive culture sensitivity testing for Klebsiella isolates. This finding is consistent with the study done by Mettler et al and Maraha et al. ${ }^{21}$ The mean duration of hospital stay in empirical sensitive therapy group and empirical resistance group were $8.87 \pm 4.51$ days and $11.89 \pm 5.10$ days respectively. So, there is highly significant difference $(\mathrm{P}<0.0001)$ between two treatment arms on decreasing duration of hospital stay (unpaired t-test) which shows better clinical outcome. Study performed by Eiland III et al showed similar results. ${ }^{22}$

For the prevention of development of resistance antimicrobial stewardship programme should be initiated in the hospital and guidelines should be formulated for effective use of antimicrobials at hospital. Antimicrobial stewardship programme have been shown to reduce inappropriate antimicrobial use, with resulting reductions in antimicrobial resistance, and also lead to more appropriate antimicrobial therapy for infections where therapy is required, with improved clinical outcomes for patients. ${ }^{23}$ Solutions for antimicrobial resistance will not be easy and, paradoxically increasing the price of antibiotics might restrict their use, but delicate balance between overuse and lack of access is to be maintained. ${ }^{24}$

\section{CONCLUSION}

Initial empirical therapy with broad-spectrum antimicrobials is a treatment strategy for severe Klebsiella infections. With an awareness of local pathogen prevalence and resistance profiles, as well as a consideration of patient clinical characteristics, the physician can implement an initial empirical antimicrobial regimen that is likely to be active against the probable causative pathogen. Thereby decreasing the risk of death and the potential for complications and longer hospital stay (and associated costs) that are associated with inappropriate empirical therapy. Modification of broad-spectrum coverage when the causative pathogen is identified decreases the duration of broad-spectrum antimicrobial exposure, minimizes the potential for the emergence of resistance, and provides additional cost savings.

Funding: No funding sources Conflict of interest: None declared

Ethical approval: The study was approved by the Institutional Ethics Committee 


\section{REFERENCES}

1. World Health Organization. World Health Organization Report on Infectious Disease. Overcoming Antimicrobial Resistance. The World Health Report 2000. Geneva: World Health Organization; 2000.

2. Gumbo T. General Principles of Antimicrobial Therapy. In: Brunton L, editor. Goodman \& Gilman's The Pharmacological Basis of Therapeutics. 13th Ed. New York: McGraw Hill; 2011.957-968.

3. Johnson A. Surveillance of antibiotic resistance. Philosophical Transactions of the Royal Society B: Biol Sci. 2015;370(1670).

4. Culture Media. In: Ananthnarayan R, Panikar CKJ. Textbook of Microbiology. 8th Edition. Hyderabad: Universities Press; 2009;34.

5. John JF Jr, Fishman NO. Programmatic Role of the Infectious Diseases Physician in Controlling Antimicrobial Costs in the Hospital. Clin Infect Dis 1997;24:471-85.

6. Chiu J, Thompson GW, Austin TW, Hussain Z, John M, Bombassaro AM et al. Antibiotic Prescribing Practices for Catheter Urine Culture Results. Can J Hosp Pharm. 2013;66 (1):13-20.

7. Berild D, Mohseni A, Diep LM, Jensenius M, Ringertz SH. Adjustment of antibiotic treatment according to the results of blood cultures leads to decreased antibiotic use and costs. J Antimicrob Chemother. 2006;57:326-30.

8. Rakesh A. Antimicrobial sensitivity pattern of Klebsiella pneumonia isolated from pus from tertiary care hospital and issues related to the rational selection of antimicrobials. J. Chem. Pharm. Res. 2013; 5(11):326-31.

9. Santana R, Gaspar G. Secular trends in Klebsiella pneumoniae isolated in a tertiary-care hospital: increasing prevalence and accelerated decline in antimicrobial susceptibility. Revista da Sociedade Brasileira de Medicina Tropical. 2016;49(2):177-82.

10. Berild D, Mohseni A, Diep LM, Jensenius M, Ringertz SH. Adjustment of Antibiotic Treatment According to the Results of Blood Culture Leads to Decreased Antibiotic Use and Cost. J Antimicrobial Chem. 2006;57:326-30.

11. Kollef M. Broad-Spectrum Antimicrobials and the Treatment of Serious Bacterial Infections: Getting It Right Up Front. Clin Inf Diseases. 2008;47(S1):3-13.

12. Micek ST, Roubinian N, Heuring T, Bode M, Williams J, Harrison C, et al. Before-after study of a standardized hospital order set for the management of septic shock. Critical care medicine. 2006 Nov 1;34(11):2707-13.

13. Podschun R, Ullmann U. Klebsiella spp. as Nosocomial Pathogens: Epidemiology, Taxonomy, Typing Methods, and Pathogenicity Factors. Clinical Microbiol Reviews. 1998;11(4):589-603.
14. Fahmey S. Early-onset sepsis in a neonatal intensive care unit in Beni Suef, Egypt: bacterial isolates and antibiotic resistance pattern. Korean $\mathbf{J}$ Pediatr. 2013;56(8):332.

15. Paterson DL. Impact of Antibiotic Resistance in Gram-Negative Bacilli on Empirical and Definitive Antibiotic Therapy. Clinical Infectious Diseases. 2008;47:S14-20.

16. Suryawanshi S. Antibiotic Prescribing Pattern in a Tertiary Level Neonatal Intensive Care Unit. J Clin And Diagnostic Res. 2015;Vol-9(11).

17. Donovan FM, Windle ML, Herchline TE. Community Acquired Pneumonia Empiric Therapy. Available at: http://emedicine.medscape.com/article/2011819overview. Accessed 23 November 2018.

18. El Bouamri M, Arsalane L, El Kamouni Y, Zouhair S. Antimicrobial susceptibility of urinary Klebsiella pneumoniae and the emergence of carbapenemresistant strains: A retrospective study from a university hospital in Morocco, North Africa. African J Urol. 2015;21(1):36-40.

19. Park S. Third-generation cephalosporin resistance in gram-negative bacteria in the community: a growing public health concern. The Korean J Internal Medicine. 2014;29(1):27.

20. Azar SL, Ebadi AR. Examining the Pattern of Susceptibility and Antibiotic Resistance in Klebsiella pneumoniae Strains Isolated from Urine Samples of Children with Urinary Tract Infections from the Children's Hospital of Tabriz in 2015. British Biomedical Bulletin. 2017;(5)307.

21. Mettler J, Simcock M, Sendi P, Widmer AF, Bingisser R, Battegay $M$ et al. Empirical Use of Antibiotics and Adjustment of Empirical Antibiotic Therapies in a University Hospital: a Prospective Observational Study. BMC Infectious Diseases. 2007;7:21.

22. Eiland EH, Beyda N, Han J, Lindgren W, Ward R, English TM, et al. The Utility of Rapid Microbiological and Molecular Techniques in Optimizing Antimicrobial Therapy. SRX Pharmacol, vol. 2010, Article ID 395215,2010;6.

23. SARI. A Strategy for the Control of Antimicrobial Resistance in Ireland. Available online at: https://www.hpsc.ie/AZ/

MicrobiologyAntimicrobialResistance/InfectionContr olandHAI/Guidelines/File,41 16,en.pdf. Accessed on: 23 November 2018.

24. Laxminarayan R, Duse A, Wattal C, Zaidi AKM, Wertheim HFL, Sumpradit N, et al. Antibiotic Resistance-The Need for Global Solutions. The Lancet Infectious Diseases. 2013;13(12):1057-98.

Cite this article as: Damor RR, Kubavat AR. Antibiotic sensitivity profile of Klebsiella isolates \& it's impact on clinical outcome. Int J Basic Clin Pharmacol 2019;8:1819-24. 